\title{
Science Opportunities at the SwissFEL X-ray Laser
}

\author{
Bruce D. Patterson ${ }^{\mathrm{a}}$, Paul Beaud ${ }^{\mathrm{a}}$, Hans H. Braun ${ }^{\mathrm{a}}$, Catherine Dejoie ${ }^{\mathrm{ab}}$, Gerhard Ingold ${ }^{\mathrm{a}}$, Christopher \\ Milne $^{a}$, Luc Patthey ${ }^{a}$, Bill Pedrinia ${ }^{\text {, Jakub Szlachentko }}{ }^{a}$, and Rafael Abela*a
}

\begin{abstract}
Next-generation X-ray sources, based on the X-ray Free Electron Laser (XFEL) concept, will provide highly coherent, ultrashort pulses of soft and hard X-rays with peak intensity many orders of magnitude higher than that of a synchrotron. These pulses will allow studies of femtosecond dynamics at nanometer resolution and with chemical selectivity. They will produce diffraction images of organic and inorganic nanostructures without deleterious effects of radiation damage.
\end{abstract}

Keywords: Cross correlations · Free electron laser - Photochemistry · Pump and probe experiments · Single shot spectroscopy $\cdot$ X-ray diffraction

\section{Introduction}

Several laboratories around the world are currently constructing and developing Free Electron Lasers (FELs), currently the world's brightest sources of UV and X-ray radiation. The research applications of the ultra-short and intense pulses produced by these sources range from condensed matter and materials research, to femto-chemistry, molecular biology, atomic spectroscopy, and many more.

Existing facilities, FLASH ${ }^{[1]}$ at DESY in Hamburg, Germany, LCLS ${ }^{[2]}$ at Stanford, USA, FERMI ${ }^{[3]}$ in Trieste, Italy and SACLA ${ }^{[4]}$ in Hyogo, Japan, as well as the upcoming X-ray FELs SwissFEL in Switzerland, European XFEL ${ }^{[5]}$ in Hamburg, Germany and PAL-XFEL in Pohang, South Korea, all provide or will provide photon pulses of duration less than $100 \mathrm{fs}$ and with a peak brilliance many orders of magnitude beyond that of conventional third-generation synchrotron light sources.

The Paul Scherrer Institute is building the SwissFEL, which is expected to begin user operation in the year 2017. The facility consists of a high-brightness electron gun, a $5.8 \mathrm{GeV}$ linear accelerator, perma- nent magnetic undulators and a variety of photon beamlines to several experimental stations.

The SwissFEL (Fig. 1) uses a lowemittance electron beam in conjunction with short-period in-vacuum undulators to generate hard X-rays at the relatively low electron energy of $5.8 \mathrm{GeV}$. These specifications can be realized with a relatively short, $700 \mathrm{~m}$, facility. The final concept foresees two undulator lines for different wavelength regions. The first beamline to be built, Aramis, is designed for wavelengths between 0.1 and $0.7 \mathrm{~nm}$; Table 1 lists the corresponding X-ray pulse design

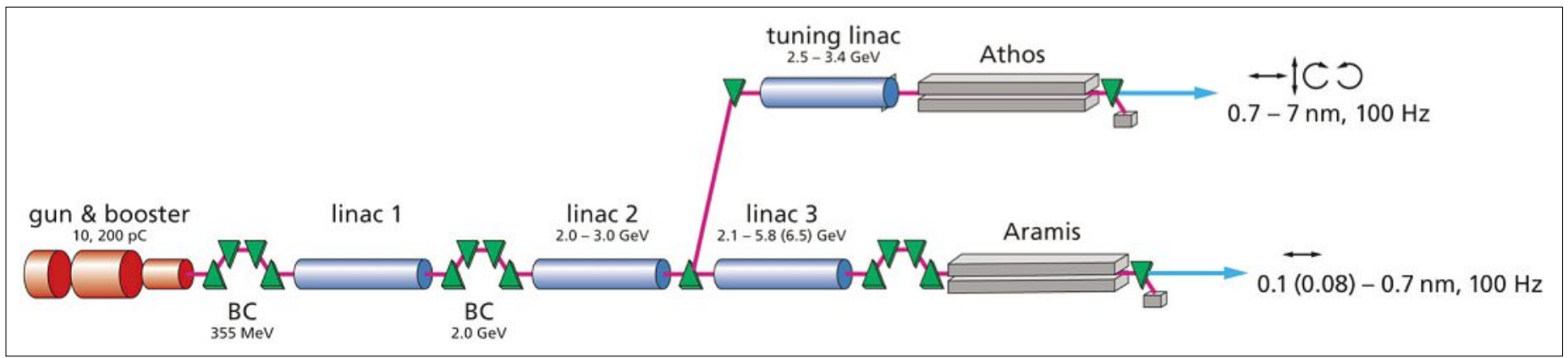

Fig. 1. Schematic plan of the SwissFEL facility, including the hard and soft X-ray beamlines Aramis and Athos (undulators in grey).

Table 1. Design parameters for various operating modes of the SwissFEL Aramis photon beam, at $1 \AA ̊$ wavelength

\begin{tabular}{|c|c|c|c|}
\hline \multirow[t]{2}{*}{ Parameter } & \multicolumn{2}{|c|}{ Nominal Operation Mode } & \multirow{2}{*}{$\begin{array}{c}\text { Upgrade } \\
\text { Ultra-Short Pulses }\end{array}$} \\
\hline & Long Pulses & Short Pulses & \\
\hline pulse energy $[\mu \mathrm{J}]$ & 60 & 3 & 6 \\
\hline peak power [GW] & 2 & 0.6 & 11 \\
\hline RMS pulse length [fs] & 13 & 2.1 & 0.3 \\
\hline bandwidth [\%] & 0.03 & 0.04 & 0.05 \\
\hline $\begin{array}{l}\text { peak brightness [photons / } \\
\left.\left(\mathrm{mm}^{2} \mathrm{mrad}^{2} \mathrm{~s} 0.1 \% \text { bw }\right)\right]\end{array}$ & $3 \cdot 10^{32}$ & $1 \cdot 10^{32}$ & $1.3 \cdot 10^{33}$ \\
\hline repetition rate $[\mathrm{Hz}]$ & 100 & 100 & 100 \\
\hline
\end{tabular}

\footnotetext{
${ }^{\star}$ Correspondence: Dr. R. Abela ${ }^{a}$

Tel. +4156 3103271

E-mail: rafael.abela@psi.ch

aSwissFEL

Paul Scherrer Institute

OVGA 413

$\mathrm{CH}-5232$ Villigen-PSI

bLaboratorium für Kristallographie

ETH Zürich

$\mathrm{CH}-8093$ Zürich
}

repetition rate $[\mathrm{Hz}]$ 
parameters. The second undulator beam line, Athos, is designed for wavelengths in the range $0.6 \mathrm{~nm}$ to $7 \mathrm{~nm}$, and will offer the option of circularly-polarized radiation. By virtue of the acceleration of double electron bunches and fast-switching magnets, X-ray pulses will be simultaneously available at both Aramis and Athos with a $100 \mathrm{~Hz}$ repetition rate.

Specialties foreseen for the SwissFEL facility include: nearly transform-limited 'self-seeded' pulses, a mode with $4 \%$ FWHM 'broad-bandwidth' wavelength range, a pump-probe time resolution of approximately 10 fs FWHM, optics, instrumentation and detectors for the strategically interesting photon energy range 2-4 keV, flexible options for sample excitation with non-ionizing terahertz pulses, and in-house developed two-dimensional (2D) X-ray detectors with single-photon sensitivity, large dynamic range and virtually zero dark noise. Two hard X-ray experimental stations are presently being planned for ultrafast, photo-pumped investigations at Aramis: ESA for liquid-phase chemistry and biochemistry and ESB for crystalline bulk and thin-film materials.

\section{Science Opportunities}

\subsection{Photochemistry and Biology 2.1.1 Conceptual Design}

Experimental Station A (ESA) is envisioned as a pump-probe X-ray spectroscopy station, ${ }^{[6]}$ for investigating processes in biology and chemistry that can be triggered with light. ESA will have three significant advantages, which it plans to exploit:

i) There is significant expertise in Switzerland in time-resolved X-ray absorption and emission spectroscopy, on both solid and liquid samples, providing a strong local base of expert users.

ii) SwissFEL will generate $\mathrm{X}$-rays in the range from $2-5 \mathrm{keV}$, which is a photon energy range that is difficult to access at the facilities commonly used by the XAS/ XES community. This energy range includes elemental absorption edges of $\mathrm{P}, \mathrm{Cl}$, $\mathrm{S}, \mathrm{K}$, and $\mathrm{Ca}$, which are relevant to biology, and of Ti, which is relevant to both sustainable energy sources and photocatalysis. By building a dedicated in-vacuum X-ray spectrometer for this energy range, ESA will provide to scientists a unique tool not available at other X-ray sources.

iii) SwissFEL will have the ability to tune the photon energy easily courtesy of the variable-gap undulator design, which greatly facilitates X-ray spectroscopy measurements.

Although ESA is focused on providing scientists with easy access to the 2-5 $\mathrm{keV} \mathrm{X}$-ray energy range, the beamline design allows the full energy range of
SwissFEL (1.7-12.4 keV) to be used for both monochromatic $(0.01-0.03 \%)$ and broad bandwidth (3-4\%) experiments with achromatic X-ray focusing down to $1 \mu \mathrm{m}$ spot size. By combining this flexible and powerful photon delivery ability with the Paul Scherrer Institute's expertise in X-ray detector development the following techniques will be feasible at ESA:

- pump-probe X-ray spectroscopy, including X-ray absorption (XAS), $\mathrm{X}$-ray emission (XES), and Resonant Inelastic X-ray Scattering (RIXS)

- pump-probe X-ray diffuse scattering

- femtosecond serial nanocrystallography, both pump-probe and steady state

- pump-probe X-ray diffraction on molecular crystals

It must be emphasized that this list of techniques should not be taken to imply that other methods will not be feasible at ESA. The goal of the experimental station is to allow users to implement their own measurements with maximum flexibility.

The simplest X-ray spectroscopic technique to perform is X-ray absorption spectroscopy, where the X-ray signal from the sample, measured either in transmission or fluorescence, is monitored as a function of the incident X-ray photon energy. The resulting spectrum contains a wealth of electronic and structural information and the technique can be applied to a broad range of disordered samples, ranging from solids, to liquids, to gases. This type of measurement was recently performed at the LCLS hard X-ray FEL in Stanford (USA). An ultrafast spin photo-excitation in a molecular system was probed using XAS around the Fe K-edge at $7.1 \mathrm{keV} .^{[7]}$ By measuring the XAS signal both without and with laser excitation as a function of the time delay between pump (laser) and probe (X-rays), they were able to resolve the transient XAS signal of the molecule in its high-spin state on the femtosecond timescale. These measurements clearly demonstrate the ability to perform time-resolved XAS at FEL facilities with femtosecond time resolution (Fig. 2).

After an X-ray absorption process has taken place a core hole is present in the absorbing atom, this core hole can be filled in several different ways, e.g. through relaxation of a higher-lying electron, causing the sample to emit either an electron or a photon. The remaining relaxation processes involve production of electrons, primarily through Auger decay. X-ray emission spectroscopy (XES) consists of resolving the energies of the emitted X-ray fluorescence after an absorption process has taken place. This energy-resolved spectrum contains information on the electronic and spin state of the absorbing species. ${ }^{[8-11]}$ This technique probes the occupied electronic states in the sample, in contrast to XAS which probes the unoccupied states. Using an XES spectrometer it is possible to observe the relaxation of valence electrons into the core hole, which contain information on the chemical bonds formed by the absorbing atom. In molecular orbital terms these signals probe the highest-occupied molecular orbitals (HOMO), and in band structure terminology they probe the valence band. Recently at LCLS this type of XES measurement was combined with a nanocrystallography experiment on Photosystem II, which is one of the lightharvesting centers in chlorophyll. In this experiment the sample was exposed to light and the X-ray diffraction pattern was recorded from the flowing liquid jet sample of nanocrystals simultaneously with the Mn K-edge XES signal using a dispersive $\mathrm{X}$-ray spectrometer. This combination of measurements allowed the researchers to ensure that the Mn atom at the center of the active site of the protein was undamaged while monitoring the structural changes due to the photoexcitation. ${ }^{[12]}$

It has recently been shown that by tuning the incident $\mathrm{X}$-ray photon energy just below the absorption edge it is possible to

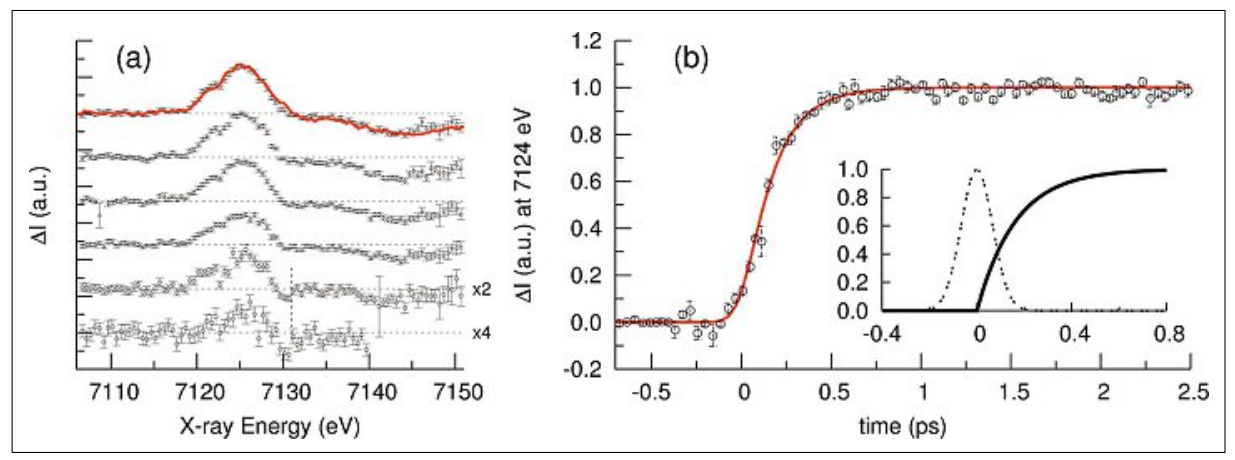

Fig. 2. a) Transient $X A S$ measured at different time delays between laser pump and $X$-ray probe $(-170,0,190,370,500$ and $1250 \mathrm{fs})$ from bottom to top on an aqueous solution of $\left[\mathrm{Fe}(\mathrm{bpy})_{3}\right]^{2+}(\mathrm{b})$ Time scan measured at one energy showing the difference signal, fitted with an exponential rise time of $163 \pm 6$ fs convolved with an instrument response of $155 \pm 6$ fs. From ref. [7]. Reprinted (adapted) with permission from H. T. Lemke et al., J. Phys. Chem. A 2013, 117, 735. Copyright (2013) American Chemical Society. 
measure a scattering signal that contains information on the X-ray absorption spectrum. ${ }^{[13]}$ This experiment, named high-energy resolution off-resonant spectroscopy (HEROS), can be performed in a singleshot technique with the same technical requirements as for XES measurements.

The idea of using off-resonant excitations dates back to 1982 when, following the Kramers-Heisenberg relation, ${ }^{[14]}$ Tulkki and Åberg developed simplified formulas to describe the second-order photon-atom interaction. ${ }^{[15]}$ This theory is often used to calculate both the resonant and non-resonant $\mathrm{X}$-ray emission spectra around the $\mathrm{ab}$ sorption edge of a scattering atom. ${ }^{[16-19]}$ In their original work, Tulkki and Åberg noticed that for incident beam energies tuned below an X-ray absorption edge (called the off-resonance region), the shape of the $\mathrm{X}$-ray emission spectrum is proportional to the unoccupied density of states of an atom. However until recently, the potential of extracting the electronic structure from a single X-ray emission spectrum recorded at off-resonant excitation energies was not explored, likely due to the extremely weak scattering cross section as compared to that determining the resonant X-ray emission spectrum. ${ }^{[18]}$ By combining off-resonant excitation and an X-ray spectrometer operating in a dispersive geometry at a synchrotron, HEROS has been experimentally demonstrated. ${ }^{[13,20]}$ An example of HEROS is shown in Fig. 3. HEROS is an alternative to XAS, with significant advantages when used with pulsed X-ray sources. By taking
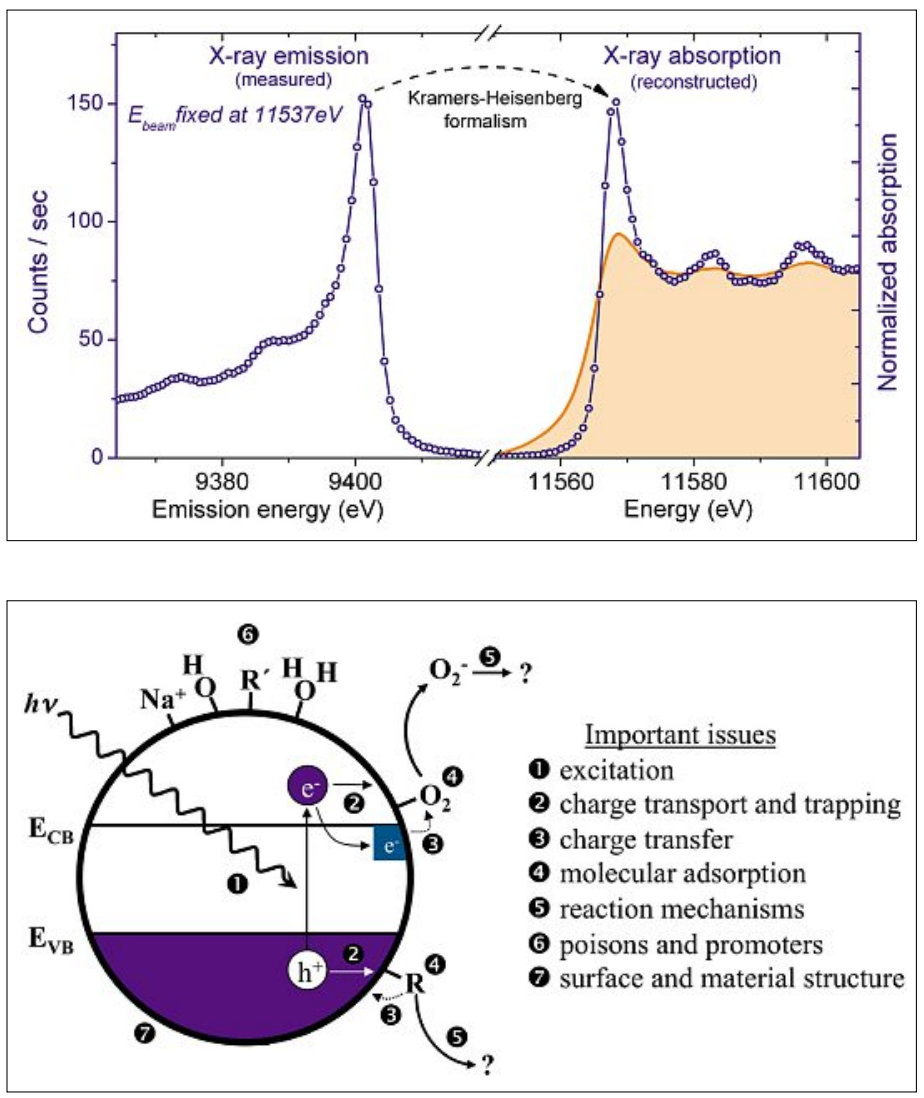

Fig. 4. Diagram showing issues involved with electronic excitation of nanoparticles and the subsequent reactions that can occur. From ref. [21]. Reprinted from M. A. Henderson, Surface Science Reports, 2011, 66, 185. Copyright (2011), with permission from Elsevier. and relaxation processes of the electronhole pair created upon excitation of the nanoparticle with UV light and to identify the trapping mechanism on an ultrashort timescale $(<1 \mathrm{ps})$.

By taking advantage of ESA's ability to perform a variety of measurements concurrently, we will be able to perform XAS, XES, and X-ray diffraction on these nanoparticles. XAS provides details on the unoccupied electronic states of the semiconductor conduction band, as well as on the local electronic and geometric structure around the Ti atoms in the nanocrystals. XES provides information on the occupied states of the semiconductor valence band. X-ray spectroscopy can thus provide detailed information on the electronic states involved in the electron-hole pair formation, and their evolution in time after photo-excitation. As a complement to the spectroscopic measurements ESA will also be capable of measuring the wide-angle X-ray scattering signal (WAXS) from the nanocrystals, which provides information on the crystal lattice dynamics after photoexcitation. By measuring the lattice dynamics and correlating them with the spectroscopic measurements of the electronic relaxation on the femtosecond timescale it will be possible to establish how electronphonon coupling affects the formation of the long-lived trapped electronic states.

\subsection{Pump and Probe in Condensed Matter \\ 2.2.1 Conceptual Design}

The proposed station ESB dedicated to perform pump and probe experiments in condensed matter combines time-resolved laser spectroscopic methods and X-ray scattering techniques to study the dynamics of cooperative interactions in crystalline materials that exhibit long-range electronic and magnetic order. Important representatives are the strongly correlated electron systems or 'quantum materials' that exhibit competition between lattice, charge, orbital and spin degrees of freedom. From the correlations of the atomic, electronic and magnetic constituents new phases or states of condensed matter emerge. Recent examples include materials with high-temperature superconductivity, colossal magnetoresistance, metal-toinsulator transition, electron fractionalization and novel quantum-critical states. Multiferroics, materials combining several functional properties, hold promise for future device applications. To characterize and control such materials - especially when they are not in strict thermodynamic equilibrium - is ideally done in the time domain where the coupled excitations can be distinguished on account of their different response and relaxation times. Photon-in/photon-out scattering allows to 
directly correlate the electronic, magnetic and structural dynamics.

At the hard X-ray ARAMIS beamline we propose to install an instrument for $\mathrm{X}$-ray pump-probe (XPP) scattering and diffraction with polarization control and flexible sample environment. It includes a fs laser system, X-ray optics, diagnostics and three experimental setups. In a first phase, (i) a general purpose heavy load sample stage with robot detector arm and cryo XPP chamber and (ii) a heavy load diffractometer with detector arm including polarization analysis for elastic scattering at high magnetic fields will be installed. Once ARAMIS self-seeding becomes available, (ii) a compact medium energy resolution spectrometer for inelastic scattering at high pressure will be installed.

Novel quantum phases in solids often emerge via coupling between the lattice, charge, orbital and spin systems exhibiting long-range order. To experimentally study such systems the measured quantity must be allowed to infer information about the spectroscopic properties of fermionic (quasiparticle) excitations, about the dynamic properties of bosonic (collective) modes, and about the correlations embedded in the two-particle properties of the large number of interacting constituents. Such information can be inferred from the dynamic structure factor $S(q, \omega)$ which is measured in X-ray scattering.

Resonant and non-resonant X-ray diffraction (coherent elastic scattering) can measure long-range atomic, electronic and magnetic order whereas elastic and inelastic (incoherent) X-ray scattering are sensitive to both thermal and non-thermal (quantum mechanical) fluctuations.

At SwissFEL, employing these techniques in the time-domain using $\mathrm{X}$-rays in the range $4-12.4 \mathrm{keV}$, elementary excitations can be probed which dictate the response of the system when it is driven both near to and far from equilibrium with an optical laser or THz pump-pulse. To study electronically and spin driven dynamics in the time domain, XPP measurements with time resolution well below 50 fs have to be performed. Main areas of investigation include:

- (Coherent) dynamics and coupling in non-equilibrium systems;

- Correlations and fluctuations in nonequilibrium phase transitions;

- Real-time evolution of electronic correlations;

- Selective excitation of electronic, spin and structural order;

- Coupling, control and switching in multiferroics;

- Non-linear transient response in condensed matter systems;

- (Quantum) control of response functions.

\subsubsection{Applications}

As an example we briefly discuss a new class of experiments foreseen at endstation ESB, namely to employ diffuse scattering in the time-domain to study electronically induced criticality in correlated electron systems ('quantum materials'). Critical systems are close to a critical point, generally identified as the boundary of an order-disorder phase transition; they can avoid to be trapped in one of the two extreme states: the long-range ordered state in which the interactions are too strong and the constituents are locked, and the disordered state in which the interactions are too weak and the system is dominated by noise. Second-order phase transitions (e.g. a ferromagnetic transition) exhibit criticality when a control parameter (e.g. temperature) drives the system from one phase to another across the critical point. Close to the critical point, fluctuations exhibiting spatial and temporal correlations develop which give rise to power law distributions (critical exponents) in certain thermodynamic quantities that describe the response of the system (e.g. magnetic susceptibility). Fluctuations can be driven thermally or non-thermally. Ultimately it should be possible to measure stimulated criticality of materials that exhibit a quantum critical point (QCP) - a zero-temperature phase transition - separating an ordered from a quantum-disordered state (i.e. one caused by quantum fluctuations) which can be controlled by a magnetic field or by pressure.

Employing time-domain diffuse scattering, it has recently been demonstrated that stimulated density-density correlations can be measured at a hard X-ray free electron laser. ${ }^{[23]}$ In this novel experiment the phonon-phonon correlations of germanium have been measured to access large portions of the phonon dispersion.

For phonons, the correlation function $\left.\mathrm{g}(\mathrm{t})=<\mathrm{u}_{\mathrm{q}}(0) \mathrm{u}_{-\mathrm{q}}\right\}(\mathrm{t})>$ is the variance of atom displacements with amplitude $u_{\mathrm{q}}$. Upon excitation with a femtosecond laser pulse the time evolution of the variance $\mathrm{g}(\tau)$ of correlated pairs of phonons at reduced wavevector $\mathrm{q}$ and $-\mathrm{q}$ is measured stroboscopically as a function of the pump-probe delay $\tau$ between the optical pulse and the X-ray pulse. As shown in Fig. 5, the evolution of $g(\tau)$ derived from the change of the normalized diffuse scattering intensity has an oscillatory contribution which is attributed to a two-phonon squeezed state. Squeezing arises if the system is initially in a timeindependent eigenstate (or admixture of eigenstates). The optical excitation of the electronic system with an ultrashort laser pulse causes a fast change of the atomic potential and hence a 'softening' of optical and acoustic phonon branches over a large subset of the Brillouin zone. For a harmonic atomic potential, the sudden decrease in the frequency of the harmonic oscillator will 'squeeze' the position-space distribution of the oscillator and launch an oscillation in the expectation value of the variance of the position which is twice the frequency of the normal mode after excitation. This oscillation in the quadrature of the normal-mode coordinate (and its corresponding canonical momentum) is what is meant by 'phonon squeezing'. It is shown in Fig. 5 for two different reduced wavevectors $\mathrm{q}$. The phonon dispersion $\omega(\mathrm{q})$ is then obtained by applying the Fourier transform.

In a previous experiment ${ }^{[24]}$ we studied phonon squeezing by exploiting the Debye-Waller effect in Bragg diffraction to measure the time-dependence of the mean-square displacements of atoms in pure bismuth that is excited with a laser pulse (see Fig. 6). Now these types of studies have been extended to time-resolved $\mathrm{X}$-ray diffuse scattering. This method is more general because correlations (variances) caused by atomic displacements and also by charge or spin disorder ${ }^{[25]}$ can be measured for a particular wavevector throughout the Brillouin zone.

One class of experiments in the future would cover charge (CDW) and spin (SDW) density wave systems close to a QCP at high pressure where a THz pumppulse selectively excites vibrational modes ('phonon pumping') which couple to the CDW or SDW system.

\section{Pilot Experiments in Structure Determination}

\subsection{Using a Non-monochromatic Microbeam for Serial Snapshot Crystallography}

The SwissFEL facility will provide a broad-bandpass mode with an energy bandwidth of about $4 \%$, and this has im-

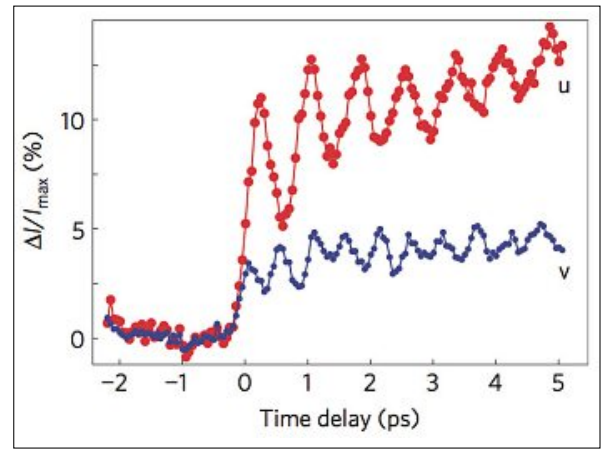

Fig. 5. Femtosecond X-ray diffuse scattering. Change in normalized diffuse scattering intensity $\Delta \mathrm{l}(\tau) / \mathrm{I}_{\max } \alpha \mathrm{g}(\tau)$ due to phonon squeezing for two different reduced wavevectors $q$ in the (022) Brillouin zone of germanium as a function of (optical)pump-(X-ray)probe delay. From ref. [20]. 


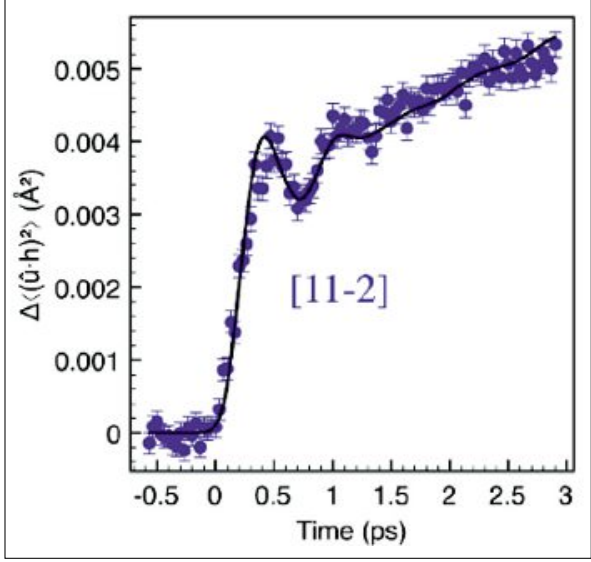

Fig. 6. Femtosecond X-ray diffraction. Timeresolved atomic position variance measured at the (11-2) diffraction peak of bismuth where phonon squeezing causes a decrease in the magnitude of diffraction via the Debye-Waller factor. The solid line shows a fit to a simple model of phonon squeezing. From ref. [21]. Reprinted from M. A. Henderson, Surface Science Reports, 2011, 66, 185. Copyright (2011), with permission from Elsevier.

portant implications for structural studies. By exposing a small crystallite (from nano- to a few micrometers in size) to a single ultrafast pulse, a diffraction pattern can be obtained before the crystal is damaged. If such single-pulse diffraction patterns, collected sequentially on many randomly oriented crystallites, are combined, it is possible to determine the structure of the material accurately. ${ }^{[26]}$ Performing such an experiment with strictly monochoromatic beam has a serious drawback: only a single position of the Ewald sphere is accessed in each pattern, and, because reflections have a finite width, the diffraction condition is not satisfied completely for any of the reflections recorded. By using the $4 \%$ energy range of the SwissFEL beam, a new option for structural studies of crystalline materials may become possible. The use of such an 'extra pink' beam in a diffraction experiment with stationary crystallites should not only increase the number of reflection intensities that can be collected in a single shot, but also overcome the problem of 'partial reflection' measurement that is inherent to the monochromatic experiment.

In a recent proof-of-concept study, a new approach, inspired by the Laue single-crystal (micro)diffraction technique was examined. ${ }^{[27]}$ Diffraction patterns for 100 randomly oriented stationary crystallites of the MFI-type zeolite ZSM-5 were simulated assuming several energy bandwidth values and two detector positions. These necessarily sparse diffraction patterns could be indexed using a pattern recognition algorithm. The number of accessible reflections that are not affected by the partial reflection intensity problem increases significantly with bandwidth.
Furthermore, the number of reflection intensities that can be measured in a single shot is also significantly higher than with monochromatic radiation. On average, with a $4 \%(0.5 \%)$ bandwidth, there are 140 (17) reflections per pattern with a $2 \mathrm{D} 1 \mathrm{M}$ Pilatus detector positioned at $90^{\circ}(2 \theta)$ relative to the incident beam and 46 (6) reflections with the detector at $45^{\circ}(2 \theta)$. For 100 randomly oriented patterns, a completeness value of $c a .65 \%$ was calculated for each individual setup, with an increase up to $80 \%$ (0.9-2.5 A resolution range) for the two setups combined. Structure solution, using either direct methods or a dual-space method, with the reflections from these patterns was successful for bandwidth values greater than $3 \%$.

In the near future the SwissFEL experiment will be simulated at synchrotron sources. With the real data obtained there problems of intensity correction, indexing and scaling will be tackled. This new approach could be of benefit to the protein community as well as in the area of inorganic and small molecule structures, where the diffraction patterns are much sparser. The 'extra pink' beam mode option at SwissFEL also offers a clear opportunity to ease the data acquisition and subsequent evaluation in femtosecond time-resolved experiments.

\subsection{Two-dimensional Structure from Random Multiparticle X-ray Scattering Images Using Cross- correlations}

The cross-correlation method proposed already in 1977 by Kam ${ }^{[28]}$ allows three dimensional (3D) structural information of a particle to be accessed by accumulating data extracted from a large set of (noisy) scattering images on single- or multiparticle random configurations. The procedure is the subject of renewed interest because X-ray Free Electron Lasers may provide the required photon flux. Below, we describe procedure and outcome of an analogous 2D pilot experiment, simplified to be feasible at a synchrotron source. It resulted in the reconstruction of the $2 \mathrm{D}$ electron density of a $350 \mathrm{~nm}$ sized gold nanoparticle.

In Coherent X-ray Diffraction Imaging (CDI) the 2D electron density of an object can be reconstructed from its scattering image with a phasing algorithm, provided that the image in reciprocal space is sufficiently oversampled and that sufficient signal to noise is achieved. The crosscorrelation method finds its application if such conditions cannot be met, which typically happens when only random multiparticle scattering images can be acquired. The key point is the accumulation of data from different images to properly evaluate the cross-correlations. In 2D, the scatter- ing pattern of a single particle can then be unambiguously determined.

For the 2D cross-correlation test experiments, a large number of identical, effectively 2D gold nanostructures were grown in random orientation on a membrane. This was scanned through the X-ray beam at the cSAXS beamline of the Swiss Light Source, so that each time a different multiparticle arrangement was illuminated. Fig. $7 \mathrm{a}$ is an example of the 3751 diffraction recorded images, originating from about 20 nanoparticles. Fig. $7 \mathrm{~b}$ is the $2 \mathrm{D}$ diffraction image of the single particle reconstructed following the cross-correlation based protocol, described in detail in ref. [29].
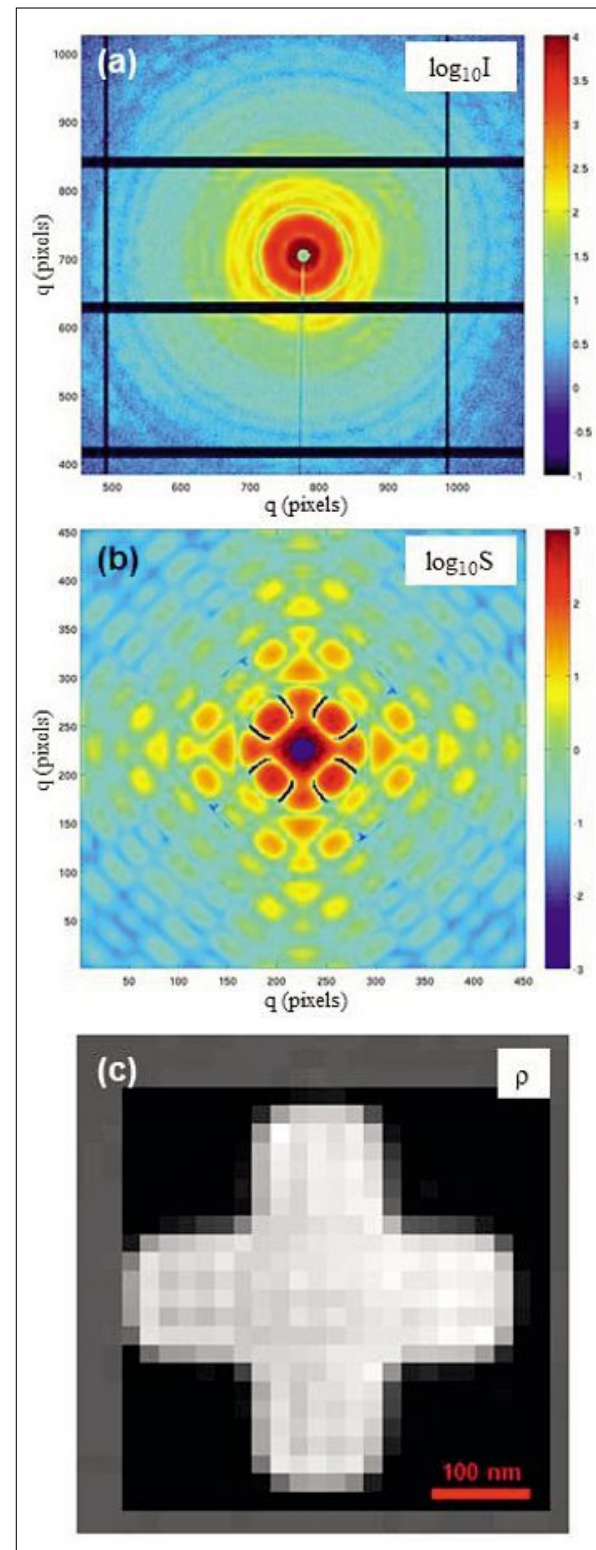

Fig. 7. (a) Example of a multi-particle diffraction image. The measured number of photon counts $\mathrm{S}$ is displayed in logarithmic scale. (b) Single-particle diffraction pattern $S(q, \phi)$, reconstructed using the cross-correlation method from 3751 multi-particle diffraction images of which (a) is a typical example. (c) 2D charge density $\rho$, obtained by applying a phasing algorithm to the data of (b). 
Finally, Fig. 7c displays the reconstructed 2D electron density of the particle, and represents a first convincing proof of principle of 2D structure determination based on the cross-correlation method. The upcoming challenge is to execute the experiment in $3 \mathrm{D}$, which is accompanied by practical and computational complications. Extension to particles in solution will require a sufficient number of scattered photons within the rotational correlation time of the particles. It is thus evident to consider the FEL as the $\mathrm{X}$-ray radiation source.

\section{Outlook}

Progress is continuing on the realization of several XFEL projects worldwide: the LCLS in California and the SACLA facility in Japan are producing prodigious amounts of spectacular data and the interest in XFELs of the scientific community is rapidly growing. The construction of SwissFEL is progressing well; operation in Villigen-Würenlingen can begin in 2017. The Paul Scherrer Institute welcomes input from all interested parties.

\section{Acknowledgements}

The authors acknowledge the efficient and enjoyable cooperation within the SwissFEL project team, the strong support of the PSI research divisions and the thoughtful input of university colleagues. Part of the work was supported by the NCCR-MUST. The Advanced Light Source at the Lawrence Berkeley National Laboratory is supported by the Office of Science, Office of Basic Energy Sciences, Scientific User Facilities Division of the US Department of Energy under contract No. DE-AC02-05CH11231.

Received: November 25, 2013

[1] For latest information on FLASH, see: http:// flash.desy.del

[2] For latest information on LCLS, please see: http://lcls.slac.stanford.edu/

[3] For latest information on FERMI, please see: http://www.elettra.trieste.it/FERMI/
[4] For latest information on SACLA, please see: http://xfel.riken.jp/

[5] For latest information on European XFEL, please see: http://www.xfel.eu/

[6] SwissFEL Experimental Station A: Conceptual Design Report ESA, PSI report, June 2013.

[7] H. T. Lemke, C. Bressler, L. X. Chen, D. M. Fritz, K. J. Gaffney, A. Galler, W. Gawelda, K. Haldrup, R. W. Hartsock, H. Ihee, J. Kim, K. H. Kim, J. H. Lee, M. M. Nielsen, A. B. Stickrath, W. Zhang, D. Zhu, M. Cammarata, J. Phys. Chem. A 2013, 117, 735 .

[8] G. Vanko, T. Neisius, G. Molnar, F. Renz, S. Karpati, A. Shukla, F. M. F. de Groot, J. Phys. Chem. B 2006, 110, 11647.

[9] U. Bergmann, P. Glatzel, Photosynth. Res. 2009, 102, 255 .

[10] P. Glatzel, M. Sikora, G. Smolentsev, M. Fernandez-Garcia, M. Hard, Catal. Today 2009, $145,294$.

[11] P. Glatzel, M. Sikora, S. G. Eeckhout, O. V. Safonova, G. Smolentsev, G. Pirngruber, J. A. van Bokhoven, J.-D. Grunewaldt, M. Tromp, Synchr. Rad. Instr. 2007, 879, 1731.

[12] J. Kern, R. Alonso-Mori, R. Tran, J. Hattne, R. J. Gildea, N. Echols, C. Glöckner, J. Hellmich, H. Laksmono, R.G. Sierra, B. Lassalle-Kaiser, S. Koroidov, A. Lampe, G. Han, S. Gul, D. DiFiore, D. Milathianaki, A. R. Fry, A. Miahnahri, D. W. Schafer, M. Messerschmidt, M. Marvin Seibert, J. E. Koglin, D. Sokaras, T.-C. Weng, J. Sellberg, M. J. Latimer, R. W. Grosse-Kunstleve, P. H. Zwart, W. E. White, P. Glatzel, P. D. Adams, M. J. Bogan, G. J. Williams, S. Boutet, J. Messinger, A. Zouni, N. K. Sauter, V. K. Yachandra, U. Bergmann, J. Yano, Science 2013, 340, 491.

[13] J. Szlachetko, M. Nachtegaal, J. Sá, J.-C. Dousse, J. Hoszowska, E. Kleymenov, M. Janousch, O. V. Safonova, C. König, J. A. van Bokhoven, Chem. Commun. 2012, 48, 10898.

[14] H. A. Kramers, W. Heisenberg, Zeits. Phys. 1925, 31,681 .

[15] J. Tulkki, T. Aberg, J. Phys. B 1982, 15, L435.

[16] J. Tulkki, Phys. Rev. A 1983, 27, 3375

[17] J. A. Carlisle, E. L. Shirley, E. A. Hudson, L. J. Terminello, T. A. Callcott, J. J. Jia, D. L. Ederer, R. C. C. Perera, F. J. Himpsel, Phys. Rev. Lett. 1995, 74, 1234 .

[18] H. Hayashi, Y. Udagawa, W. A. Caliebe, C. C. Kao, Chem. Phys. Lett. 2003, 371, 125.

[19] J. Szlachetko, J.-Cl. Dousse, J. Hoszowska, M. Pajek, R. Barrett, M. Berset, K. Fennane, A. Kubala-Kukus, M. Szlachetko, Phys. Rev. Lett. 2006, 97, 073001.

[20] M. Kavčič, M. Žitnik, K. Bučar, A. Mihelič, B. Marolt, J. Szlachetko, P. Glatzel, K. Kvashnina, Phys. Rev. B 2013, 87, 075106.
[21] M. Hoffmann, S. Martin, W. Choi, D. Bahnemann, Chem. Rev. 1995, 95, 69; M. A. Henderson, Surface Sci. Rep. 2011, 66, 185.

[22] N. Vlachopoulos, P. Liska, J. Augustynski, M. Gratzel, J. Am. Chem. Soc. 1988, 110, 1216.

[23] M. Trigo, M. Fuchs, J. Chen, M. P. Jiang, M. Cammarata, S. Fahy, D. M. Fritz, K. Gaffney, S. Ghimire, A. Higginbotham, S. L. Johnson, M. E. Kozina, J. Larsson, H. Lembke, A. M. Lindenberg, G. Ndabashimiye, F. Quirin, K. Sokolowski-Tinten, C. Uher, G. Wang, J. S. Wark, D. Zhu, D. A. Reis, Nature Phys. Advance Online Publication, www.nature.com/ naturephysics, 2013.

[24] S. L. Johnson, P. Beaud, E. Vorobeva, C. J. Milne, E. D. Murray, S. Fahy, G. Ingold, Phys. Rev. Lett. 2009, 102, 175503.

[25] Y. Feng, J. Wang, R. Jaramillo, J. van Wezel, S. Haravifard, G. Srajer, Y. Liu, Z.-A. Xu, P.B. Littlewood, T. F. Rosenbaum, Proc. Natl. Acad. Sci. USA 2012, 109, 7224; S. Haravifard, A. Banerjee, J. C. Lang, G. Srajer, D. M. Silevitch, B. D. Gaulin, H. A. Dabkowska, T. F. Rosenblum, Proc. Natl. Acad. Sci. USA 2012, 109, 2286.

[26] H. N. Chapman, P. Fromme, A. Barty, T.A. White, R. A. Kirian, A. Aquila, M. S. Hunter, J. Schulz1, D. P. DePonte, U. Weierstall, R. B. Doak, F. R. N. C. Maia, A. V. Martin, I. Schlichting, L. Lomb, N. Coppola, R. L. Shoeman, S. W. Epp, R. Hartmann, D. Rolles, A. Rudenko, L. Foucar, N. Kimmel, G. Weidenspointner, P. Holl, M. Liang, M. Barthelmess, C. Caleman, S. Boutet, M. J. Bogan, J. Krzywinski, Ch. Bostedt, S. Bajt, L. Gumprecht, B. Rudek, B. Erk, C. Schmidt, A. Hömke, Ch. Reich., D. Pietschner, L. Strüder, G. Hauser, H., J. Ullrich, S. Herrmann, G. Schaller, F. Schopper, H. Soltau, K.-U. Kühne, M. Messerschmidt, J. D. Bozek, S. P. Hau-Riege, M. Frank, Ch. Y. Hampton, R.G. Sierra, D. Starodub, G. J.Williams, J. Hajdu, N. Timneanu, M. M. Seibert, J. Andreasson, A. Rocker, O. Jönsson, M. Svenda, S. Stern, K. Nass, R. Andritschke, C.-D. Schröter, F. Krasniqi, M. Bott, K. E. Schmid, X. Wang, I. Grotjohann, J. M. Holton, T. R. M. Barends, R. Neutze, S. Marchesini, R. Fromme, S. Schorb, D. Rupp, M. Adolph, T. Gorkhover, I. Andersson, H. Hirsemann, G. Potdevin, H. Graafsma, B. Nilsson and J. C. H. Spence, Nature 2011, 470, 73 .

[27] C. Dejoie, L. B. McCusker, C. Baerlocher, R. Abela, B. D. Patterson, M. Kunz, N. Tamura, $J$. Appl. Cryst. 2013, 46, 791.

[28] Z. Kam, Macromolecules 1977, 10, 927.

[29] B. Pedrini, A. Menzel, M. Guizar-Sicarios, V. A. Guzenko, S. Gorelick, C. David, B. D. Patterson, R. Abela, Nature Comm. 2013, 1647. 